Res., Soc. Dev. 2019; 8(6):e20861042

ISSN 2525-3409 | DOI: http://dx.doi.org/10.33448/rsd-v8i6.1042

\title{
Prevalência e aspectos epidemiológicos de enteroparasitoses em crianças no Brasil
}

Prevalence and epidemiological aspects of enteroparasitoses in children in Brazil

Prevalencia y aspectos epidemiológicos de la enteroparasitosa en niños en Brasil

Recebido: 11/03/2019 | Revisado: 18/03/2019 | Aceito: 24/03/2019 | Publicado: 29/03/2019

Thiago das Virgens Santos

ORCID: https://orcid.org/0000-0003-4128-8875

Universidade Federal Vale do São Francisco, Brasil.

E-mail: santosvirgens@ hotmail.com

Rita de Cassia Macêdo Santos

ORCID: https://orcid.org/0000-0003-0724-0894

União Metropolitana de Educação e Cultura, Brasil.

E-mail: ritauesc@ hotmail.com

Victor Hugo da Silva Martins

ORCID: https://orcid.org/0000-0002-0103-9332

Universidade Federal Vale do São Francisco, Brasil.

E-mail: victorugow@hotmail.com

Sarah Alves Martins

ORCID: https://orcid.org/0000-0003-1985-8560

Universidade Católica de Salvador, Brasil.

E-mail:ssarahmartins@hotmail.com

Nadyr Cristina Bezerra

ORCID: https://orcid.org/0000-0003-3317-419X

Universidade Federal Vale do São Francisco, Brasil.

E-mail: nadyr-uni@hotmail.com

\section{Resumo}

Vivemos em um mundo tecnológico e repleto de constantes mudanças, o que não é distinto quando se trata de saúde. As enteroparasitoses são um problema grave e que tem afetado a população, principalmente os cidadãos que não possuem condições higiênico-sanitárias adequadas. O objetivo deste artigo é buscar evidências disponíveis na literatura sobre a prevalência de aspectos epidemiológicos de enteroparasitoses em crianças no Brasil. O estudo foi realizado por meio de revisão bibliográfica, através de levantamento de artigos científicos 
utilizando as principais bases de indexação científicas, tais como: SciELO, MedLine e outros. A prevalência de enteroparositose no Brasil é elevada, estando os problemas sociais e o acesso parcial as políticas públicas como colaboradores na propagação de enfermidades, dentre elas as parasitoses intestinais, do qual ocupam o topo. As políticas públicas de saúde devem ser inseridas e desfrutadas pela comunidade desassistida nesses locais, visto que as crianças em virtude de suas condições fisiológicas, são mais sensíveis a tais manifestações.

Palavras-chave: Perfil Epidemiológico; Doenças Parasitárias; Verminoses; Condutas Terapêuticas; Parasitologia.

\begin{abstract}
We live in a technological world and full of constant changes, which is not distinctive when it comes to health. The enteroparasitoses are a serious problem and that has affected the population, mainly the citizens who do not have adequate hygienic-sanitary conditions. The objective of this article is to search for available evidence in the literature on the prevalence of epidemiological aspects of enteroparasitosis in children in Brazil. The study was carried out by means of a bibliographical review, through a survey of scientific articles using the main bases of scientific indexing, such as: Scielo and MedLine. The prevalence of enteroparositosis in Brazil is high, with social problems and partial access to public policies as collaborators in the spread of diseases, among them intestinal parasitoses, of which they occupy the top. Public health policies should be inserted and enjoyed by the unassisted community in these places, since children by virtue of their physiological conditions are more sensitive to such manifestations.
\end{abstract}

Keywords: Epidemiological Profile; Parasitic diseases; Verminoses; Therapeutic Conduct; Parasitology.

\title{
Resumen
}

Vivimos en un mundo tecnológico y repleto de constantes cambios, lo que no es distinto cuando se trata de salud. Las enteroparasitosas son un problema grave y que ha afectado a la población, principalmente a los ciudadanos que no tienen condiciones higiénico-sanitarias adecuadas. El objetivo de este artículo es buscar evidencias disponibles en la literatura sobre la prevalencia de aspectos epidemiológicos de enteroparasitos en niños en Brasil. El estudio fue realizado por medio de revisión bibliográfica, a través del levantamiento de artículos científicos utilizando las principales bases de indexación científicas, tales como: Scielo y MedLine. La prevalencia de enteroparositosis en Brasil es elevada, estando los problemas 
sociales y el acceso parcial a las políticas públicas como colaboradores en la propagación de enfermedades, entre ellas las parasitosis intestinales, del cual ocupan la cima. Las políticas públicas de salud deben ser insertadas y disfrutadas por la comunidad desasistida en esos lugares, ya que los niños en virtud de sus condiciones fisiológicas, son más sensibles a tales manifestaciones.

Palabras clave: Perfil Epidemiológico; Enfermedades Parasitarias; Gusanos; Conductos Terapéuticos; Parasitología.

\section{Introdução}

As parasitoses intestinais representam um fator preocupante para a saúde pública no Brasil, assim como em outros países em desenvolvimento, sendo amplamente distribuídas. Estima-se que cerca de 3,5 bilhões de pessoas sofram dessa condição mundialmente. No Brasil, milhões de habitantes são acometidos por alguma espécie, muitas dessas pessoas na maioria das vezes encontram-se ou vivem em locais de risco social, com condições de vida insalubres. Por certo, quando as crianças são parasitadas, necessitam de uma maior atenção, principalmente aquelas em que a carência alimentar e hábitos familiares de cuidado pessoal deficiente estão associados (Soares, Oliveira \& Souza, 2018).

As crianças são as mais acometidas, com consequências importantes, como deficiência nutricional, logo que algumas espécies espoliam elementos essenciais ao crescimento e maturação celular, ocasionando um prejuízo no crescimento e desenvolvimento. Dentre os fatores de risco social em que o indivíduo está conectado, pode-se considerar que a falta de saneamento básico, características socioeconômicas, educacionais, consumo de água sem tratamento e a contaminação de alimentos estão associado a diversas causas de infecção, principalmente as enteroparasitoses (Gurgel et al., 2005; Benitez et al., 2016).

Enteroparasitoses são infecções intestinais causadas por parasitas, podendo estes ser helmintos ou protozoário. É uma doença que afeta todas as faixas etárias, sendo mais predominante em crianças. Os danos que os enteroparasitas podem causar em pessoas acometidas incluem: obstrução intestinal (Ascaris lumbricoides), a desnutrição (Ascaris lumbricoides e Trichuristrichiura), anemia por deficiência de ferro (Ancilostomídeos) e quadros de diarréia e de má absorção (Entamoebahistolytica e Giardiaduodenalis) (Iasbik et al., 2018).

As doenças parasitárias, em sua maioria, são clinicamente assintomáticas, entretanto, os sintomas mais comuns da infecção por parasitas são diarreia, anemia, desnutrição e baixo 
peso, sendo infecções oportunistas, aproveitando do estado fisiológico do indivíduo (Streck \& Salvador, 2018). Assim, faz-se de grande importância a investigação das enteroparasitoses no Brasil, portanto, o objetivo deste artigo é buscar evidências disponíveis na literatura sobre a prevalência de aspectos epidemiológicos de enteroparasitoses em crianças no país.

\section{Metodologia}

Trata-se de um estudo com coleta de dados realizada a partir de fontes secundárias, por meio do levantamento bibliográfico por ocasião da realização de uma revisão integrativa. Todos os artigos analisados fazem alusão ao saneamento básico, falta de água tratada e nível de instrução deficiente com determinantes no processo de evolução de diversas doenças em especial as parasitoses, já que estão intimamente ligadas a essas condições sociais.

A revisão da integrativa é um método que tem como finalidade agrupar resultados de pesquisas sobre um determinado tema ou questão, de maneira sistemática, ordenada e abrangente. Proporcionando a incorporação de evidências sobre um tópico, no intuito de contribuir para o aprofundamento do conhecimento investigado (Mendes, Silveira \& Galvão, 2018).

Para o levantamento dos artigos, a busca foi conduzida por meio da base de dados Biblioteca Virtual da Saúde (BVS), a partir de artigos disponíveis na Scientific Electronic Library Online (SciELO) e na National Library of Medicine (MedLine).

Foram utilizados os seguintes descritores e suas combinações nas línguas portuguesa, espanhola e inglesa: "Parasitose", "Desigualdade Social", "Prevalência em crianças". Como critérios de inclusão determinaram-se: trabalhos de acesso livre na íntegra, em formato de artigo científico, publicados nos idiomas português, inglês e espanhol, no período de 2005 a 2018. Tanto à análise quanto à síntese dos dados extraídos dos artigos foram realizadas de forma descritiva, permitindo notar, contar, descrever e classificar os dados, com o objetivo de agrupar o conhecimento sobre o tema abordado nesta revisão.

\section{Resultados e discussão}

Encontrou-se 1.416 referências nas bases de dados, sendo: SciELO - 1.374 artigos e MedLine - 42 artigos. Avaliado 32 referências na íntegra, sendo excluídos dois artigos por não responderem à questão norteadora. Assim, no total foram incluídas 29 referências nesta revisão integrativa. 
As enteroparasitoses são doenças causada por helmintos ou protozoários podendo provocar diversas alterações patológicas a depender da espécie do parasita, sendo essas: obstrução intestinal, anemia por espoliação de elementos essenciais e desnutrição em consequência da quantidade insuficiente de minerais/vitaminas para a construção de macromoléculas. E para se ter um bom prognóstico é necessário a adoção de condutas que insiram tanto o parasitado, como as pessoas da família que mantém contato frequente, já que a ausência de hábitos salutares no grupo familiar, são propagadores da enfermidade (Ely et al., 2011).

E uma das complicações grave é o óbito, principalmente em crianças em idade escolar. No mundo corresponde por cerca de 3 milhões de mortes, sendo as crianças as mais acometidas, e isso se deve a sua fragilidade imunológica, a dependência de cuidados de terceiros e ao contexto social em que está inserida (Lima, Santos, Siqueira, Medeiros Filho \& Pontes Filho, 2016).

No Brasil, embora se tenha registrado uma tendência na redução da prevalência das infecções por parasitos intestinais por conta das campanhas educacionais, com orientações para o uso de água tratada, hábitos de vida saudáveis e o acesso a medicamentos, ainda observa-se persistência dessas doenças em algumas regiões do país, em especial na região norte e nordeste, onde as pessoas estão em maior risco social, com precários condições de vida e acesso parcial as políticas públicas de saúde em analogia a outras regiões do país, em que os indivíduos possuem maior acesso a diversos serviços, situação que pode ser justificada pelo alto nível de instrução educacional (Bacelar, Santos, Monteiro, Calegar, Nascimento \& Carvalho-Costa, 2018).

$\mathrm{O}$ aumento da migração da população rural para os áreas urbanizadas em busca de melhores condições de vida, proporcionou um crescimento dos aglomerados no centros urbanos, favorecendo as chances de exposição a diversas doenças, em especial as parasitoses, haja vista que, condições socio sanitárias e o contexto de vida são determinantes na disseminação, logo que algumas práticas de autocuidado são negligenciadas. Ademais, situações precárias de vida, como o acesso a alimentos sem a devida higienização e o desenvolvimento socioeconômico deficiente da população constituem aspectos amplificadores que facilitam a propagação do problema, uma vez que favorecem a manutenção dos ciclos de contaminação de vários agentes patogênicos, bem como a transmissão a diversas pessoas (Barbosa, 2013; Oliveira, 2013). 
As crianças em idade escolar são as mais susceptíveis, pois representam um grupo de alto risco para infecções por parasitos transmitidos através do solo, da água e do ar, já que estão em um período de intenso crescimento físico e rápido metabolismo, resultando em necessidades nutricionais aumentadas, sendo o organismo um local ideal para o crescimento e maturação do parasita, pois a oferta de elementos vitais é constante e abundante (Silva, Lima, Castro \& Sousa, 2015).

As parasitoses intestinais no Brasil acometem principalmente a população de baixa renda, sem acesso ao saneamento básico, à assistência médica e às informações adequadas sobre medidas profiláticas. Tal situação é comum em todas as cidades brasileiras, sendo mais notável nos bairros periféricos e cidades distantes dos polos industriais. Mas elas, tem relação estreita com o nível educacional e cuidado em saúde, sendo considerada uma infecção democrática e restrita a um público com deficiências em acessar bens básicos, tanto de saúde, como de saneamento. As hortaliças e água não tratada, representam importantes meios de transmissão de enteroparasitoses, uma vez que helmintos e protozoários podem estar presentes nas mesmas, por meio de material fecal de origem humana, ou ainda contaminação do solo por uso de adubo orgânico com dejetos fecais (Espíndola, 2014; Abreu, Andrade, Machado \& Persoli, 2016).

Em estudo, foram verificados a presença de formas parasitárias de helmintos e protozoários, como: oocistos de Cryptosporidium sp.; cistos de Giárdia sp., Entamoeba sp. e Balantidium coli, ovos de Ascarídideos e Ancylostoma e Strongyloides em água no rio Marombas situado na porção central do estado de Santa Catarina, região Sul do Brasil, sendo um ponto preocupante, logo que muitas família fazem uso da água para atividades domesticas e consumo. Já em outra pesquisa realizado na região nordeste do país em comunidade rural, verificou-se uma prevalência dos parasitas Ascaris lumbricoides, Entamoeba coli e Giardia duodenalis, que podem causar obstrução intestinal e desconforto gastrointestinal, sendo prevalente em pessoas que consumiam produtos de feiras livres, sem a devida higienização anteriormente (Cordeiro et al., 2014; Souza et al., 2016).

A maturação insuficiente do sistema imunológico de crianças e a dependência de cuidados, deixa esse grupo etário mais vulnerável a agravos de qualquer espécie, especialmente as enteroparasitoses, já que uma das vias de contaminação é a fecal-oral e elas dependem de terceiros quanto a alimentação e outros recursos básicos de higiene (Vasconcelos, Oliveira, Cabral, Coutinho \& Menezes, 2011).

Além disso, famílias de baixa classe social e que vivem em áreas com más condições de higiene coletiva e pessoal, estão entre as mais suscetíveis para infecção por parasitas 
intestinais, de modo que diversos aspectos da vida do indivíduo tem determinação no processo de doença, estando a criança um organismo ativo nessas etapas (Santos, Souza, Frizzo, Mallet \& Pedroso, 2013).

A mortalidade em menores é reflexo das condições socioambientais de uma área e o acesso aos serviços básicos de saúde. E dependendo do agente causador da doença, algumas medidas preventivas devem ser adotadas na terapêutica e orientações quanto a mudanças de hábitos culturais. Podemos verificar esse fato com a observação da taxa de hospitalidade no ano de 2015 em crianças menores de 9 anos nas regiões do Brasil, oriunda por helmintíase, da qual somam 423 internações (Baptista, Silva Ramos \& Santos, 2014; Brasil, 2015).

A região que detém maior percentual dessas internações causadas por helmintos é o Nordeste com um total de 160 (38\%) hospitalizações, seguido pelo Sudeste 121(29\%), Norte 67(16\%), Sul 60(14\%), e Centro-Oeste 15(3\%). As infecções parasitárias induzem aos frequentes quadros de anemia e isso pode ser explicado pela ação espoliativa de que alguns parasitos exercem sobre seus hospedeiros de elementos fundamentais, como ferro e vitaminas, importantes componentes para a formação e estruturação celular (Santos, Souza, Frizzo, Mallet \& Pedroso, 2013).

Certamente um dos fatores para essa quantidade de hospitalizações nas regiões nordeste e sudeste, está ligado ao saneamento básico e consumo de água tratada, inexistente ou ineficaz em determinadas pontos. No Nordeste a cobertura de saneamento básico e água as residências é bem inferior, em analogia a região sul. O Brasil promove o acesso de água, ainda em uma realidade distante, locais periféricos ainda acessam com diversas dificuldades. Cerca de 35 milhões de brasileiros, não tem acesso a esse serviço, estando a maior parcela concentrada principalmente no norte e nordeste do país (Araújo, Silva Filho, Silva, Filha, Nogueira, 2016).

Em relação ao saneamento básico a região Sudeste possui $77,4 \%$ de cobertura por redes de esgoto, enquanto as regiões Nordeste, Sul e Norte têm, respectivamente, 27\%, 25,9\% e $4 \%$ de cobertura. A água pode ser veículo para a transmissão de inúmeras doenças, pois, além de conter a presença de bactérias e dos vírus, a água serve também como vetor para inúmeros protozoários e vermes que podem provocar efeitos patológicos, não somente no ser humano, mas também em muitos animais (Barreto, Pedreira \& Will, 2015; Sousa \& Bocardi, 2015).

Ainda conforme a base de dados do Ministério da Saúde, o Brasil no ano de 2015 notificou uma morte por helmintíase em crianças menores de 9 anos. Esse óbito aconteceu na região nordeste do país. Contudo, as parasitoses intestinais não constituem risco imediato de 
morte na infância, a sua relação com a diarreia e a desnutrição pode colocar em risco a sobrevivência da criança, em virtude de uma eventual desidratação e comprometimento de órgãos (Lima, Santos, Siqueira, Medeiros Filho, Pontes Filho, 2016).

O risco de mortalidade por doenças diarreicas é maior entre as crianças menores de um ano, devendo-se evitar a desidratação e manter um aporte adequado de nutrientes que garantam uma recuperação mais rápida, recomendando o uso de soro caseiro e a procura por um serviço médico o mais precocemente possível, para ser adotada conduta adequada (Sá, 2016).

Consequentemente a transmissão fecal-oral é a forma que ocorre com maior frequência, em especial entre as crianças, sendo a água e os alimentos contaminados seus principais veículos. A manipulação do alimento sem os devidos cuidados antes, durante e após o preparo, torna-o um propagador da infecção parasitaria. Porém, existem fatores que favorecem a elevada prevalência dos parasitos em ambientes fechados, entre os quais se destacam a facilidade de contato (criança-criança/criança-adulto) e os hábitos higiênicos das crianças e dos adultos (Brito, Melo, Araújo, Brito \& Madi, 2013; Barbosa, 2013).

Portanto, a implantação de práticas educativas que conduzam as pessoas a adquirir conhecimentos sobre as parasitoses, as tornam capazes de atuarem na prevenção e redução da carga parasitária, sendo uma estratégia eficaz e eficiente em todos os ambientes. Nesse sentido, para o controle das enteroparasitoses é necessário mudar o comportamento da população em especial as que estão em maior risco. Dentre algumas medidas estão: tratamento adequado de água, tratamento do doente e das pessoas da família para evitar o ciclo de contaminações, lavar as mãos antes das refeições ou manusear alimentos e evitar coçar a região anal desnuda e levar a mão após a região oral (Rocha, Coelho, Santos \& Silva, 2013; Amaro, 2016).

A conscientização da população sobre bons hábitos de higiene, realização de exame parasitológico periódico e o tratamento de indivíduos com diagnostico confirmado e os assintomáticos representam uma importante estratégia para prevenção de parasitoses e a diminuição da propagação, já que a disseminação é relacionada com alguns hábitos. E devido a correria durante o dia de algumas pessoas que trabalham distantes de casa, o consumo de alimentos em estabelecimentos sem o conhecimento prévio do preparo torna-se uma problemática, sendo necessário também a oferta de orientações aos colaboradores de ambientes que fornecem comida (Cunha, Silva, Carvalho \& Platino, 2016).

De fato, a investigação parasitológica por meio de inquéritos socioambientais e a busca ativa de pacientes parasitados pode consistir em uma importante ferramenta para 
auxiliar no melhor direcionamento das políticas públicas de Saúde e redução dos casos em determinadas localidades. A prevenção de várias doenças e relacionada ao nível educacional e o investimento em educação para a saúde são primordiais, devendo iniciar nas escolas, afim de que penetrem nos seios familiares, quebrando ciclos nocivos de doenças facilmente evitáveis (Azevedo, Ribeiro, Correia, Soares, Rocha \& Alves, 2013).

\section{Conclusão}

A prevalência de enteroparositoses em crianças no Brasil é elevada, sendo os problemas sociais enfrentados pelas pessoas de baixa renda, como colaboradores na propagação de enfermidades. O estímulo de práticas de prevenção e autocuidado, em ambiente doméstico e escolar, são uteis para que a quantidade de casos de enteroparasitoses seja atenuada.

Essa temática necessita de maior atenção principalmente nos centros com pouca visibilidade e em localidades periféricas da sociedade onde as condições de vida são degradantes. As políticas públicas de saúde devem ser inseridas e desfrutadas pela comunidade desassistida nesses locais no intuito de diminuir os números de hospitalizações por causas evitáveis.

\section{Referências}

Abreu, E. S., Andrade, M. B. L., Machado, Á., \& Persoli, L. B. L. (2016). Análise da qualidade parasitológica de alfaces orgânicas vendidas em uma rede de supermercados da cidade de São Paulo. Revista da Universidade Vale do Rio Verde, 14(2), 516-521.

Amaro, G. T. (2016). A abordagem educativa para prevenção das parasitoses intestinais. Disponível em: https://ares.unasus.gov.br/acervo/handle/ARES/3547. Acesso em: mar 2019.

Araújo, S. C., Silva Filho, J. A., Silva, G. M. S., Filha, M. C., \& Nogueira, V. F. B. (2016). Distribuição espacial de indicadores operacionais de serviço de abastecimento de água no Nordeste Brasileiro. Revista Verde de Agroecologia e Desenvolvimento Sustentavel, 11(1), 20-28. 
Azevedo, M. C. P. A., Ribeiro, D. F., Correia, B. R., Soares, A. K. F., Rocha, M. K. L., \& Alves, E. R. P. (2013). Educação em saúde: uma ferramenta para a prevenção e controle de parasitoses. Revista da Universidade Vale do Rio Verde, 11(2), 300-310.

Bacelar, P. A. A., Santos, J. P. D., Monteiro, K. J. L., Calegar, D. A., Nascimento, E. F. D., \& Carvalho-Costa, F. A. (2018). Parasitoses intestinais e fatores associados no estado do Piauí: uma revisão integrativa.

Baptista, A. B., da Silva Ramos, L., \& Santos, H. A. G. (2014). Prevalência de enteroparasitos e aspectos epidemiológicos de crianças e jovens do município de altamira-pa/prevalence of intestinal parasites and epidemiological aspects of children and youth in the municipality of altamira, Pará, Brazil. Revista de Pesquisa em Saúde, 14(2).

Barbosa, V. A. (2013). Educação sanitária como prática de prevenção de parasitoses intestinais em creches. Acervo da Iniciação Científica, (1).

Barreto, R. L., Pedreira, M. M., \& Will, R. M. (2015). Monitoramento da qualidade da água para consumo humano no estado da Bahia no ano 2014. Revista Baiana de Saúde Pública, 39, 31-40.

Benitez, A. D. N., Mareze, M., Miura, A. C., Brunieri, D. T. S. C., Ferreira, F. P., MitsukaBreganó, R., \& Navarro, I. T. (2016). Abordagem da saúde única na ocorrência de enteroparasitas em humanos de área urbana no norte do Paraná. Arq. ciênc. vet. zool. UNIPAR, 19(4), 203-208.

Brasil. Data, S. U. S. (2015). Ministério da Saúde-Sistema de Informações Hospitalares do SUS (SIH/SUS). Taxa mortalidade por ano processamento segundo lista morbilidade CID-10. [Interrnet]. Brasil.

Brito, A. M. G., Melo, C. M., Araújo, A. R., Brito, R. G., \& Madi, R. R. (2013). Protozoário comensal em amostra fecal: parâmetro para prevenção de infecção parasitaria via fecaloral. Scire Salutis, 3(2), 17-22. 
Cordeiro, L., Oliveira, A. T., Leite, N. K., Exterkoetter, R., Oliveira, L. J. G. G., Klein, D. \& Sá, L. S. (2014). Avaliação parasitológica das águas subterrâneas da região do rio marombas. Águas Subterrâneas.

Cunha, J. C., Silva, A. T., Carvalho, M. T. M., \& PIantino, C. B. (2016). Ocorrência de parasitoses intestinais no centro de aprendizagem pró-menor de Passos-CAPP. Revista Brasileira de Iniciação Científica, 3(4).

Ely, P. H., Balestrin, F. F., Magalhães, K. C., Dias, S. Y. F., Strada, C. B. C., \& Godoi, S. D. C. (2011). O uso do lúdico na educação em saúde: uma intervenção com crianças de uma instituição de ensino. Interbio, 5(2), 62-64.

Espíndola, C. M. D. O. (2014). Avaliação epidemiológica das parasitoses intestinais no Parque Oswaldo Cruz, Manguinhos, Rio de Janeiro, RJ (Doctoral dissertation).

Gurgel, R. Q., Cardoso, G. D. S., Silva, Â. M., Santos, L. N. D., \& Oliveira, R. C. V. D. (2005). Creche: ambiente expositor ou protetor nas infestações por parasitas intestinais em Aracaju, SE. Rev Soc Bras Med Trop, 38(3), 267-9.

Iasbik, A. F., de Arruda Pinto, P. S., Guimarães-Peixoto, R. P. M., de Oliveira Santos, T., Fernandes, F. M., da Silva, L. F., ... \& Araújo, J. V. (2018). Prevalence and transmission of intestinal parasitosis in human beings from Zona da Mata, Minas Gerais, Brazil. Bioscience Journal, 34(3).

Lima, E. Q., Santos, M. T., Siqueira, R. R., Medeiros Filho, F., \& Pontes Filho, R. N. (2016). Prevalence of intestinal parasites in the human population of the city Santa Luzia State of Paraba, Brazil. Journal of Parasitology and Vector Biology, 8(9), 86-91.

Mendes, K. D. S., Silveira, R. C. C. P., \& Galvão, C. M. (2018). Revisão integrativa: método de pesquisa para a incorporação de evidências na saúde e na enfermagem. Texto \& Contexto Enferm [serial on the internet]. 2008 Oct [cited 2011 July 26]; 17 (4): 758-64 
Oliveira, C. (2013). Frequência de enteroparasitoses em usuários do serviço público de saúde na mesorregião do Oeste Potiguar. Natal, 2013 (Doctoral dissertation, Dissertação de Mestrado-Saúde Coletiva-Universidade Estadual do Rio Grande do Norte).

Rocha, T. M., Coelho, M. D. G., Santos, A. J. U. A., \& Silva, F. A. C. (2013). Influência de fatores socioambientais na ocorrência de enteroparasitos e protozoários comensais em área periférica do município de Cristina, Minas Gerais. Revista Biociências, 19(2).

Sá, M. A. J. D. S. (2016). Educação em saúde: prevenção da diarreia aguda em crianças menores de 2 anos na Equipe Saúde da Família Geraldo Garcia 1 em Ponta Porã/MS.

Santos, C. S., Souza, P. S. A., Frizzo, M. N., Mallet, E. K. V., \& Pedroso, D. (2013). Prevalência de enteroparasitoses e sua relação com eosinofilia e anemia em pacientes do município de Santo Ângelo, Rio Grande do Sul, Brasil. Revista Saúde Integrada, 6(11-12), 293-307.

Silva, P. H. M., Lima, W. F., Castro, T. M. B. Q., \& Sousa, L. G. (2015). Contaminação do solo de áreas de recreação infantil de creches públicas por Ancylostoma sp. E Toxocara sp. em Teresina-PI. Revista Interdisciplinar, 8(4), 93-98.

Soares, A. L., Oliveira, E. A. N., \& Souza, I. F. A. C. (2018). A importância da educação sanitária no controle e prevenção ao ascaris lumbricoides na infância. Caderno de GraduaçãoCiências Biológicas e da Saúde-FACIPE, 3(3), 22.

Sousa, A. C. M., \& Bocardi, M. I. B. (2015). Hábitos de vida como fator desencadeante a parasitoses intestinais. Ideias e Inovação-Lato Sensu, 2(2), 77-92.

Souza, A. C., Alves, F. V., Guimarães, H. R., Amorim, A. C. S., Marília de Araújo, C. R. U. Z., da Silva Santos, B., ... \& Melo, A. C. F. L. (2016). Perfil epidemiológico das parasitoses intestinais e avaliação dos fatores de risco em indivíduos residentes em um assentamento rural do nordeste brasileiro. Revista Conexão UEPG, 12(1), 26-37.

Streck, E. L., \& Salvador, S. (2018). Parasitoses em crianças: uma revisão bibliográfica dos casos na America Latina. Inova Saúde, 6(2), 88-97. 
Porcentagem de contribuição de cada autor no manuscrito

Thiago das Virgens Santos - 20\%

Rita de Cassia Macêdo Santos - 20\%

Victor Hugo da Silva Martins - 20\%

Sarah Alves Martins - 20\%

Nadyr Cristina Bezerra - 20\% 